\title{
Verzeichnis der Kreise der DDR
}

1. LIEFERUNG

BEZIRK ROSTOCK [Westhälfte]

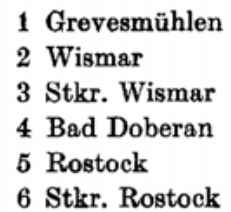

27 Magdeburg

28 Schönebeck

29 Wanzleben

30 Staßfurt

31 Oschersleben

32 Halberstadt

33 Wernigerode

34 Havelberg

35 Genthin

36 Burg

37 Zerbst

\section{LIEFERUNG}

BEZIRK ROSTOCK [Osthälfte]

\footnotetext{
7 Gadebusch

8 Schwerin

9 Stkr. Schwerin

10 Hagenow

11 Ludwigslust

12 Perleberg

13 Parchim

14 Lübz

15 Sternberg

16 Bützow

17 Güstrow

werin
}

BEZIRK SCHWERIN

\section{BEZIRK MAGDEBURG}

\author{
18 Salzwedel \\ 19 Osterburg-Seehausen \\ 20 Stendal \\ 21 Kalbe \\ 22 Klötze \\ 23 Gardelegen \\ 24 Tangerhütte \\ 25 Haldensleben \\ 26 Wolmirstedt
}

\author{
38 Ribnitz-Damgarten \\ 39 Stralsund \\ 40 Stkr. Stralsund \\ 41 Rügen \\ 42 Grimmen \\ 43 Greifswald \\ 44 Wolgast

\section{BEZIRK NEUBRANDENBURG}




\author{
59 Angermünde \\ 60 Schwedt \\ 61 Eberswalde \\ 62 Bernau \\ 63 Bad Freienwalde \\ 64 Strausberg \\ 65 Seelow \\ 66 Fürstenwalde \\ 67 Frankfurt \\ 68 Beeskow \\ 69 Eisenhüttenstadt \\ 70 Stkr. Eisenhüttenstadt
}

\section{BEZIRK POTSDAM}

$\begin{array}{ll}71 & \text { Pritzwalk } \\ 72 & \text { Wittstock } \\ 73 & \text { Kyritz } \\ 74 & \text { Neuruppin } \\ 75 & \text { Gransee } \\ 76 & \text { Oranienburg } \\ 77 & \text { Nauen } \\ \text { 78 } & \text { Rathenow } \\ \text { 79 } & \text { Brandenburg } \\ \text { 80 } & \text { Stkr. Brandenburg } \\ \text { 81 } & \text { Potsdam } \\ \text { 82 } & \text { Stkr. Potsdam } \\ \text { 83 } & \text { Belzig } \\ \text { 84 } & \text { Jüterbog } \\ \text { 85 } & \text { Luckenwalde } \\ \text { 86 } & \text { Zossen } \\ \text { 87 } & \text { Königs Wusterhausen }\end{array}$

88

BERLIN

4. LIEFERUNG

\section{BEZIRK COTTBUS}

89 Jessen

90 Herzberg

91 Bad Liebenwerda

92 Finsterwalde

93 Luckau

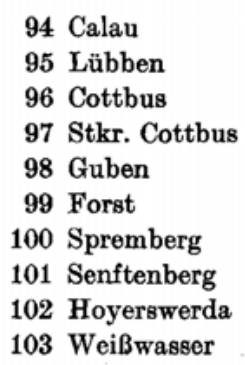

121 Rochlitz

122 Hainichen

123 Freiberg

124 Brand-Erbisdorf

125 Flöha

126 Karl-Marx-Stadt

127 Stkr. Karl-Marx-Stadt

128 Hohenstein-Ernstthal

129 Glauchau

130 Werdau

131 Reichenbach

132 Zwickau

133 Stkr. Zwickau

134 Stollberg

135 Zschopau

136 Marienberg

137 Annaberg

138 Schwarzenberg

139 Aue 
140 Auerbach

141 Klingenthal

BEZIRK GERA

142 Oelsnitz

143 Plauen

144 Stkr. Plauen

180 Jena

181 Stkr. Jena

182 Eisenberg

183 Stadtroda

184 Gera

BEZIRK LEIPZIG

185 Stkr. Gera

186 Greiz

187 Zeulenroda

188 Pößneck

189 Rudolstadt

145 Delitzsch

190 Saalfeld

191 Lobenstein

147 Stkr. Leipzig

148 Eilenburg

149 Torgau

192 Schleiz

\section{LIEFERUNG}

\section{BEZIRK HALLE}

158 Roßlau

159 Wittenberg

160 Gräfenhainichen

161 Dessau

162 Bitterfeld

163 Köthen

164 Bernburg

165 Aschersleben

166 Quedlinburg

167 Sangerhausen

168 Hettstedt

169 Eisleben

170 Saalkreis

171 Halle

172 Merseburg

173 Querfurt

174 Artern

175 Nebra

176 Weißenfels

177 Hohenmölsen

178 Zeitz

179 Naumburg
BEZIRK ERFURT

\author{
193 Nordhausen \\ 194 Worbis \\ 195 Heiligenstadt \\ 196 Mühlhausen \\ 197 Sondershausen \\ 198 Sömmerda \\ 199 Erfurt \\ 200 Stkr. Erfurt \\ 201 Langensalza \\ 202 Eisenach \\ 203 Gotha \\ 204 Arnstadt \\ 205 Weimar \\ 206 Stkr. Weimar \\ 207 Apolda
}

BEZIRK SUHL
208 Bad Salzungen

209 Schmalkalden

210 Meiningen

$211 \mathrm{Suhl}$

212 Ilmenau

213 Hildburghausen

214 Neuhaus

215 Sonneberg 